\title{
Cryptococcosis: Current status
}

DENNIS J. KITZ, PHD

KENNETH F. BARTIZAL, PHD

DALE D. ISAAK, PHD

\begin{abstract}
An opportunistic fungal pathogen, Cryptococcus neoformans may cause severe infections of the CNS, particularly in immune-compromised patients. This review article provides a thorough description of the spectrum of clinical disease caused by this organism, as well as current methods available for diagnosis and therapy of the infection. A brief overview of current research aimed at better understanding of the specific cell populations involved in host immune response to infection is provided.
\end{abstract}

Cryptococcus neoformans, an opportunistic fungal pathogen, is most noted for its ability to cause severe infection of the CNS. Although fungal infections are not required to be reported, it is strongly suspected that cryptococcal disease occurs in appreciable numbers, and, in fact, cryptococcosis has been termed ${ }^{1}$ the "awakening giant" among the mycoses.

Numerous factors have contributed to this increased appreciation for the clinical significance of Cryptococcus, including the relative severity of cryptococcal meningitis; of an estimated 200 to 300 yearly cases in the United States, the mortality rate is more than $100 .^{2}$ Although less severe, other forms of cryptococcosis, such as subclinical respiratory infections, certainly are much more prevalent. ${ }^{3}$ Finally, the morbidity and mortality associated with cryptococcosis are most severe in predisposed patients with underlying immune defects, a population that clearly is of increasing importance in contemporary medicine.

This article focuses on the microbiology of $C$ neoformans, the spectrum of clinical disease caused by this yeast, and current methods for diagnosis and therapy of infection. A brief description of current research dealing with host immune response to this organism also is presented.

\section{Microbiology}

A yeast-like fungus, $C$ neoformans exists in an asexual yeast stage pathogenic for man or in a sexual stage (Filobasidiella neoformans) related to the rust and smut pathogens of higher plants. During the yeast cycle, $C$ neoformans reproduces by budding.
Individual cryptococci are spherical, 3.5 to $7 \gamma \mathrm{m}$ in diameter, and generally encapsulated, although the extent of encapsulation is highly variable and proportional to the degree of virulence associated with a given isolate. Based on capsular polysaccharide antigens, four serotypes (A, B, C, and D) have been described, but serotypes $\mathrm{A}$ and $\mathrm{D}$ most commonly are responsible for human infection. ${ }^{4}$

In addition to the demonstration of encapsulated budding yeast cells, laboratory identification of $C$ neoformans is dependent upon specific carbon and nitrogen assimilation tests, demonstration of urease production, and lack of mycelium production during growth on corn meal agar. ${ }^{5}$

\section{Clinical disease spectrum}

Cryptococcus can infect a number of sites in the body (Table 1), but almost all human infections are thought to originate with a pulmonary focus. Small, desiccated yeast cells associated with pigeon droppings serve as the infectious units of $C$ neoformans and gain entrance to the body via the respiratory tract following inhalation. ${ }^{6}$

\section{Pulmonary cryptococcosis}

Primary pulmonary infection in the majority of patients is asymptomatic or without specific diagnostic symptoms. Patients may experience an influenza-like illness, with cough and minimal pleuritic chest pain. The degree of lung involvement varies, depending upon the immune status of the patient and the size of the infecting dose. Infections may be unilateral or bilateral, with focal or diffuse involvement; in severe disease, there is a marked predilection for involvement of the lower lung fields. ${ }^{7}$ Both mild and severe pulmonary infections may lead to dissemination to other sites in the body. Calcification associated with cryptococcosis is rare.

\section{Disseminated cryptococcosis}

The most frequently diagnosed form of cryptococcosis involves CNS infection. Although the reasons are unclear, the predilection of this yeast for the CNS is well known. It may be that certain nutrients in CSF enhance yeast growth, or that inhibitory substances that are thought to exist in serum are lacking in $\mathrm{CSF}^{8}{ }^{8}$ 
Three forms of CNS infection are observed clinically: leptomeningitis, the most common; meningoencephalitis, an uncommon but rapidly fatal type; and cryptococcomas, localized, space-occupying lesions, which are slowly expanding intracranial masses. ${ }^{9}$ In patients with cryptococcal meningitis, symptoms such as headache appear before intracranial pressure develops or papilledema can be detected. ${ }^{10}$ Symptoms of cryptococcoma vary, but they often include headache, nausea, vomiting, mental changes, coma, and paralysis. Double vision, slurred speech, and unsteadiness also may occur. ${ }^{11}$

Cryptococcus may affect many other sites. Cutaneous and mucocutaneous lesions may be seen in some immune-compromised patients. Involvement of bone is reported in $5 \%$ to $10 \%$ of cases, and visceral involvement can occur with the formation in tissues of granulomatous, gelatinous lesions, which resemble malignant neoplasias. Visceral cryptococcosis can affect any organ in late-stage disease, but lesions are most common in the heart, testes, prostate gland, and eyes. ${ }^{12}$

Approximately $50 \%$ of patients with disseminated cryptococcosis are found to be immune-compromised because of prior treatment with adrenal corticosteroids or other immunosuppressive agents, or because of conditons such as diabetes mellitus; the remaining patients have no apparent predisposing conditions. ${ }^{13}$

\section{Diagnosis}

Diagnosis of cryptococcosis is a straightforward procedure (Table 2). It involves isolation and identification of the yeast from patient specimens, direct microscopic examination of clinical specimens, and specific serologic procedures. Isolation of Cryptococcus from the patient is accomplished readily with blood agar or Sabouraud's dextrose agar containing antibacterial antibiotics. Because some strains of $C$ neoformans are sensitive to cycloheximide, selective media containing cycloheximide (for example, Mycosel agar) should not solely be used for isolation of this organism. Isolates are identified from carbohydrate assimilation patterns and urease production. ${ }^{5}$

When fungal meningitis is suspected, CSF mixed with India ink is examined microscopically for the presence of encapsulated yeasts; the capsule of the fungus is highlighted by the negative staining effect of the ink. ${ }^{14}$ Also, the yeast may be visualized in direct mounts of sputum or urine. Methenamine silver nitrate stains will reveal the presence of cryptococci in tissue biopsy specimens.

Techniques for serologic diagnosis of cryptococcosis are well established and quite useful, particularly in cases where the fungus cannot be isolated from patients with suspected infection. Serologic approaches include the detection of capsular polysaccharide antigens and $C$ neoformans specific antibody. Cryptococcal polysaccharide antigens from patient body fluids (CSF, serum, or urine) are detected using a latex-agglutination test. ${ }^{15}$ The diagnostic accuracy is $100 \%$ when both CSF and serum are assayed for antigen in patients with suspected cryptococcal menigitis. ${ }^{1}$ The antibody titer to $C$ neoformans is determined using either an indirect fluorescent antibody test or a complement fixation test.

In addition to the diagnostic value of these serologic tests, positive prognosis can be predicted by a falling antigen titer paired with a rising antibody titer.

\section{Therapy}

Localized pulmonary lesions in noncompromised patients generally have a good prognosis and resolve spontaneously. Conversely, enlarging pulmonary lesions or lesions in patients who are known to have immune defects require antifungal therapy. In addition, localized pulmonary cryptococcal lesions have been excised to rule out tumor; these patients require prophylactic therapy because of potential hematogenous dissemination of the yeast during surgery. All CNS and other disseminated forms of disease require antifungal therapy.

Amphotericin B (Fungizone) is the standard and often the only effective treatment of cryptococcosis. Amphotericin B therapy is administered in a hospital setting with the patients carefully monitored for signs of toxicity such as impaired renal function. Intravenous infusions are done slowly at concentrations of $0.1 \mathrm{mg} . / \mathrm{mL}$, and initial dosages of $0.25 \mathrm{mg} / \mathrm{kg}$ are increased as tolerance persists. Daily dosages should not exceed $1.5 \mathrm{mg} / \mathrm{kg}$. Total cumulative dosages of up to 3 grams are administered intravenously over a 6 to 10 week period. Alternate day therapy is a substitute protocol for patients experiencing intolerance to daily therapy. For patients with prior kidney damage, in whom intravenous amphotericin B was contraindicated, direct intrathecal administration has been used for CNS infection.

Combined therapy with amphotericin B and 5fluorcytosine (Ancobon) has produced a higher cure rate with fewer relapses than amphotericin $B$ alone in some studies. ${ }^{16}$ Ancobin is available as $250 \mathrm{mg}$ and $500 \mathrm{mg}$ capsules, with a dosage of 50 to 150 $\mathrm{mg} / \mathrm{kg}$ day given at 6 hour intervals. Miconazole therapy for cryptococcosis is from 1200 to $2400 \mathrm{mg} /$ day for 3 to 12 weeks or longer. One problem with intravenous use of miconazole is that it is solubilized in polyethoxylated castor oil, which may be 
TABLE 1. CLINICAL FORMS OF CRYPTOCOCCOSIS.

Pulmonary infection

Primary, self-resolving

Progressive

Disseminated infection

CNS

Skin

Mucous membrane and skin

Bone

Viscera

the primary cause of many undesirable side effects, including phlebitis, pruritus, nausea, fever, and chills.

\section{Current research on immunity}

Natural resistance to $C$ neoformans generally is protective, and, therefore, exposure rates are thought to be much higher than disease incidence. ${ }^{1}$ Thus, the organism is recognized as an opportunistic pathogen that primarily affects patients with underlying immune defects. The nature of the protective immune mechanisms that regulate $C$ neoformans has been determined in both animal and clinical studies. Both approaches have revealed that host immune responses to this infection are complex and involve specific humoral and cell-mediated immune processes, as well as nonspecific phagocytic responses. Because an extensive review ${ }^{18}$ of experimental evidence regarding host responses to Cryptococcus is available, no attempt will be made to reiterate these findings.

The association of severe forms of cryptococcosis with underlying defects in cell-mediated immunity provides indirect evidence for the importance of this aspect of the immune system in regulating $C$ neoformans. Afflicted patients often manifest skin test anergy following challenge with cryptococcal antigens, and they also yield lymphocytes with impaired capacity to secrete migration inhibition factor and undergo lymphocyte blastogenic responses following in vitro challenge with cryptococcal antigens, as well as unrelated antigens. ${ }^{19,20}$ Direct evidence for the importance of cell-mediated immunity in regulating cryptococcal infections is derived from studies demonstrating the adoptive transfer of both specific delayed-type hypersensitivity and resistance to infection following injection of T-lymphocytes from immune animals into naive recipients. ${ }^{21}$

In addition to specific cell-mediated immunity, inflammatory responses involving phagocytic cells may have relevance in regulating cryptococci. The complement (C3b) receptor has been shown to mediate uptake of cryptococci both by human monocytes and polymorphonuclear leukocytes, ${ }^{22}$ and by murine macrophages. ${ }^{23}$ Griffin $^{24}$ has pre-

\begin{tabular}{|lr|}
\hline TABLE 2. DIAGNOSIS OF CRYPTOCOCCUS INFECTION. \\
\hline Culture & Serology \\
\hline CSF* & Detection of antigen \\
Sputum & Latex agglutination test \\
Blood & Detection of antibody \\
Urine & Indirect immunofluorescence \\
Biopsy specimens & Complement fixation test \\
& \\
\hline Also microscopic observation on India ink preparation.
\end{tabular}

sented an elegant model of host response to Cryptococcus that involves regulation of macrophage surface immunoglobulin and complement receptors ( Fc and $\mathrm{C} 3 \mathrm{~b}$ ) based on exposure of macrophages to antigen-antibody complexes or soluble factors from T-lymphocytes. In addition, soluble factors from splenic lymphocytes recently have been found ${ }^{25}$ to activate macrophage killing of intracellular cryptococci, thereby providing a possible link between the protective influences of specific cell-mediated immunity and nonspecific mononuclear phagocytes.

The importance of complement-mediated uptake of Cryptococcus by phagocytic cells in the human host has been demonstrated by studies showing depression of serum complement components, particularly C3, in patients with cryptococcal fungemia. ${ }^{26}$ Cryptococcal infection with fungemia is almost always fatal despite therapy. ${ }^{27}$; the loss of complement opsonins apparently contributes to the host's inability to contain the infection.

Despite its demonstrated role as an opsonin in promoting phagocytosis, there is little evidence that soluble antibody is involved in preventing infection. Cryptococcal polysaccharide, which is known to accumulate in the CSF and serum of patients with cryptococcosis, has been shown to depress the humoral arm of the immune response in experimental animals by reducing the number of B-lymphocytes, which secrete antibody directed against this antigen. ${ }^{28,29}$ This decrease in antibody response appears to be mediated by the activation of populations of suppressor T-lymphocytes by the polysaccharide antigen. ${ }^{30}$

Specific immunologic unresponsiveness to immunization with cryptococcal polysaccharide also has been demonstrated ${ }^{31}$ in patients who have been cured of cryptococcal meningitis. These same individuals responded normally to immunization with pneumococcal polysaccharide. One might assume from these results that a similar suppressor T-cell mechanism may be responsible for the failure to produce a strong humoral response against cryptococcal polysaccharide in these cured patients.

Research directed at better understanding of natural regulating substances, such as those produced 
by immune T-lymphocytes, and their enhancing effects on the fungicidal activity of other host cell populations eventually may provide physicians with improved methods to manage the immunecompromised patient with cryptococcosis.

\section{Summary}

Cryptococcus neoformans is an opportunistic fungous pathogen which can cause life-threatening infections, particularly of the CNS, in immune-compromised patients. Diagnosis of cryptococcal infection is routine and involves visualization of the encapsulated yeasts in patient's specimens, isolation of the yeast on agar-based media with positive identification by biochemical methods, and serological detection of cryptococcal polysaccharides for antimicrobial antibody in the patient. Therapy primarily is with Amphotericin B. Basic research currently is focused on identifying immune cell populations which mediate host response to Cryptococcus infection, and ways to enhance their fungicidal activity.

1. Kaufman L, Blumer S: Cryptococcosis: The awakening giant. Pan Am Health Org Sci Pub 1977;356:176-184.

2. Annual supplement. MMWR 1976;24(summary):1-58.

3. Ajello L: A comparative study of the pulmonary mycoses of Canada and the United States. Public Health Rep 1969;84:869-877.

4. Bennett JE, Kwon-Chung KJ, Howard DH: Epidemiologic differences among serotypes of Cryptococcus neoformans. Am $J$ Epidemiol 1977; 105:582-586.

5. Lodder J: The Yeasts. Amsterdam, North-Holland, 1970.

6. Powell KE, Dahl BA, Weeks RJ, et al: Airborne Cryptococcus neoformans: Particles from pigeon excreta compatible with alveolar deposition. $J$ Infect Dis 1972;125:412-415.

7. Littman ML, Zimmerman LE: Cryptococcosis. New York, Grune and Stratton, 1956.

8. Reiss F, Szilagyi G, Mayer E: Immunological studies of the anticryptococcal factor in normal human serum. Mycopathologia 1975;55:175178

9. Yoshikawa TT, Fujita N, Grinnell V, et al: Management of central nervous system cryptococcosis. West $J$ Med 1980;132:123-133.

10. Butler WT, Alling DW, Spickard A, et al: Diagnostic and prognostic value of clinical and laboratory findings in cryptococcal meningitis. $N$ Engl J Med 1964;270:59-67.

11. Tress B, Davis S: Computed tomography of intracerebral toruloma. Neuroradiology 1979;17:223-226.

12. Salfelder K: Cryptococcosis. In Baker RD, et al (eds): The Pathologic Anatomy of the Mycoses. Berlin, Springer-Verlag, 1971.
13. Spickard A: Diagnosis and treatment of cryptococcal disease. South Med $J$ 1973;66:26-31.

14. Rippon JW: Cryptococcosis. In Medical Mycology, the Pathogenic Fungi and the Pathogenic Actinomycetes. Philadelphia, WB Saunders Co, 1982.

15. Bloomfield N, Gordon MA, Elmendorf D, et al: Detection of Cryptococcus neoformans antigen in body fluids by latex particle agglutination. Proc Soc Exp Biol Med 1963;114:64-67.

16. Bennett JE, Dismukes WE, Duma JR, et al: A comparison of amphotericin B alone and combined with flucytocine in treatment of cryptococcal menigitis. $N$ Engl $J$ Med 1979;301:126-131

17. Morgans ME, Thomas MEM, Mackenzie DW: Successful treatment of systemic cryptococcosis with miconazole. Br Med J 1979;2:100-101

18. Fromtling RA, Shadomy HJ: Immunity to cryptococcosis: An overview. Mycopathologia 1982;77:183-190.

19. Graybill JR, Alford RH: Cell-mediated immunity in cryptococcosis. Cell Immunol 1974;14:12-21.

20. Schimpff SC, Bennett JE: Abnormalities in cell-mediated immunity in patients with Cryptococcus neoformans infection. J Allergy Clin Im. munol 1975;43:430-441.

21. Lim TS, Murphy JW: Transfer of immunity to Cryptococcus by Tenriched splenic lymphocytes from Cryptococcus neoformans- sensitized mice. Infect Immun 1980;30:5-11.

22. Davies SF, Clifford DP, Hoidal JR, et al: Opsonic requirements for the uptake of Cryptococcus neoformans by human polymorphonuclear leukocytes and monocytes. J Infect Dis 1982;145:870-874.

23. Kitz DJ, Johnson CR, Kobayashi GS, et al: Growth inhibition of Cryptococcus neoformans by cloned cultured murine macrophages. Cell $\mathrm{Im}$ munol 1984;88:489-500.

24. Griffin FM Jr: Roles of macrophage Fc and C3b receptors in phagocytosis of immunologically coated Cryptococcus neoformans. Proc Natl Acad Sci USA 1981;78:3853-3857.

25. Kitz DJ, O'Rourke SR: Lymphokine-mediated inhibition of Cryptococcus neoformans growth by murine macrophages. JAOA 1985;85:684. 26. Macher AM, Bennett JE, Gadek JE, et al: Complement depletion in crytococcal sepsis $J$ Immunol 1978;120:1686-1690.

27. Diamond RD, Bennett JE: Prognostic factors in cryptococcal meningitis. Ann Intern Med 1974;80:176-181.

28. Murphy JE, Cozad GC: Immunological unresponsiveness induced by cryptococcal polysaccharide assayed by the hemolytic plaque technique. Infect Immun 1972;5;896-901.

29. Kozel TR, Gulley WF, et al: Immune response to Cryptococcus neoformans soluble polysaccharide: Immunological unresponsiveness. Infect Immun 1979;18:701-707.

30. Murphy JW, Moorhead JW: Regulation of cell-mediated immunity in cryptococcosis: I. Induction of specific afferent T-suppressor cells by cryptococcal antigen. $J$ Immunol 1982;128:276-283.

31. Henderson DK, Bennett JE, Huber MA, et al: Long-lasting, specific immunologic unresponsiveness associated with cryptococcal meningitis. $J$ Clin Invest 1982;69:1185-1190.

From the Department of Microbiology and Immunology, Kirksville College of Osteopathic Medicine, Kirksville, Mo.

Reprint requests to Dr Kitz, Department of Biological Sciences, Southern Illinois University at Edwardsville, Edwardsville, IL 62026-1651. 